\title{
Stability of Deep Water Waves \\ Governed by the Benjamin-Ono Equation
}

\author{
E. INFELD
}

Soltan Institute for Nuclear Studies, Hoża 69, 00-681 Warsaw, Poland

\author{
AND G. RowLANDS
}

Department of Physics, University of Warwick, Coventry CV4 7AL, UK

(Received September 30, 2002)

The Benjamin-Ono equation models the dynamics of internal waves in stratified fluids of great depth. It includes an integral (Hilbert transform) term, and so stability calculations might seem difficult. We expand in both the amplitude of the nonlinear wave and the wave vector of the perturbation, assumed to be small quantities of the same order. An expression for the nonlinear dispersion relation is obtained. Nonlinear periodic Benjamin-Ono waves are stable, just as the localized, algebraic soliton solutions (Lorentzians), already known to be stable. (This also follows as a limit of our calculations.) We extend the known analogy between the Benjamin-Ono and modified Korteweg-de Vries equations.

PACS numbers: 47.20.Ky, 52.35.Py

\section{Introduction}

Some time ago, Benjamin, Davis and Acrivos, and, somewhat later, Ono, formulated an equation for the propagation of deep water waves in a stratified, incompressible fluid (for some reason known as the Benjamin-Ono rather than BDAO equation) $[1,2]$ :

$$
\frac{\partial f}{\partial t}+\alpha f \frac{\partial f}{\partial x}-\beta \frac{\partial^{2}}{\partial x^{2}} \mathcal{H}(f)=0
$$




$$
\mathcal{H}(f)=\frac{1}{\pi} \mathrm{P} \int_{-\infty}^{\infty} \frac{f\left(x^{\prime}\right)}{x-x^{\prime}} \mathrm{d} x^{\prime},
$$

where P stands for the principal part of the integral. The first two formulations were for steady propagation, the third for arbitrary time dependence as above. Here $\alpha$ and $\beta$ are constants determined by the initial density profile of the fluid. The function $f$ generates the horizontal space and time dependence of both components of the fluid velocity as follows:

$$
\begin{aligned}
V_{x} & =f(x, t) \frac{\partial \Phi(y)}{\partial y}, \\
V_{y} & =-\frac{\partial f}{\partial x} \Phi .
\end{aligned}
$$

The fluid is indeed incompressible, as $\nabla \boldsymbol{V}=0$. Here $\Phi(y)$ satisfies a linear differential equation

$$
\frac{\mathrm{d}}{\mathrm{d} y}\left[\rho_{0}(y) \frac{\mathrm{d} \Phi}{\mathrm{d} y}\right]-\frac{g}{c_{0}^{2}} \frac{\mathrm{d} \rho_{0}}{\mathrm{~d} y} \Phi=0,
$$

where $c_{0}$ is a constant and $\rho_{0}(y)$ is the initial density profile as a function of the depth, assumed known. Attention usually concentrates on the more interesting Eq. (1). Over the years, considerable progress has been made here. The equation has been demonstrated to be integrable [3, 4]. It admits $N$ soliton solutions [5-7], and these solutions are stable $[8,9]$. The evolution of a general, compact initial condition has been studied [7]. Here, we will show that exact, periodic nonlinear travelling wave solutions as found by Ono [2], are also marginally stable. (In the limit of infinite wavelength, they yield the algebraic Lorentzian soliton.)

\section{Form of the wave}

Ono found an exact, propagating wave solution to Eq. (1) [2]. It was, in slightly altered notation here,

$$
\begin{aligned}
& f_{0}(\xi)=\frac{A}{1-a \cos (k \xi)}, \quad \xi=x-\lambda t \\
& A=\frac{2 \beta^{2} k^{2}}{\alpha \lambda} \\
& a=\sqrt{1-(\beta k / \lambda)^{2}}
\end{aligned}
$$

Here $k$ is a nonlinear analogue of a wave number. These exact solutions are exceptional in that most equations of the Korteweg-de Vries ( $\mathrm{KdV}$ ) class are solved by travelling waves given by elliptic functions, known as cnoidal waves, more complicated than these (elementary functions). These last solutions (6)-(8) are easily confirmed when we use the identity $(k>0)$ : 


$$
\mathcal{H}\left(\frac{1}{1-a \cos (k \xi)}\right)=\frac{a \sin (k \xi)}{\sqrt{1-a^{2}}[1-a \cos (k \xi)]} .
$$

In what follows we will need:

$$
\begin{aligned}
& \mathcal{H}\left(\mathrm{e}^{\mathrm{i} k \xi}\right)=-\mathrm{isgn}(k) \mathrm{e}^{\mathrm{i} k \xi} \\
& \mathcal{H}(\cos (k \xi))=\operatorname{sgn}(k) \sin (k \xi) .
\end{aligned}
$$

This second part is the lowest order term of (9). In fact, (9) can conversely be derived by repeated use of $(10 \mathrm{~b})$. In the $k \rightarrow 0$ limit, the solutions $(6)-(8)$ tend to a Lorentzian

$$
\lim _{k \rightarrow 0} f_{0}(\xi)=\frac{4 \beta^{2} / \lambda}{\xi^{2}+\beta^{2} / \lambda^{2}}
$$

Directly derivable from Eq. (1) when we use

$$
\mathcal{H}\left(\frac{1}{\xi^{2}+\delta^{2}}\right)=\frac{\xi}{|\delta|\left(\xi^{2}+\delta^{2}\right)} .
$$

This solution is known to be stable. These solitons are likewise unusual for the $\mathrm{KdV}$ family, as single humped solitons usually involve hyperbolic secans functions.

In this paper we will address the problem of stability of the periodic, nonlinear waves given by (6)-(8), as well as algebraic profile structures (11). These have in fact been generated experimentally by Davis and Acrivos [1]. They were seen to propagate at a steady rate without distortion. This experimental fact might lead us to expect stability.

Incidentally, all the above solutions are virtually identical to a class of solutions to the modified $\mathrm{KdV}$ equation

$$
\frac{\partial f}{\partial t}+f^{2} \frac{\partial f}{\partial x}+\beta \frac{\partial^{3} f}{\partial x^{3}}=0
$$

which is integrable. As both the solutions and the integrability properties are similar, we might like to compare the stability properties of (1) and (12). Small amplitude, cnoidal, periodic travelling wave solutions to $(12)$ are unstable $[10,11]$. Nevertheless, the very special exact solutions treated here are stable. (For a calculation specific to this problem, see Appendix.) Solitons of (12) are also stable. We will see that the same scenario governs Eq. (1), thus strengthening the similarity of the two equations. All this might point at a deeper correspondence between these two equations. This would be interesting in view of the different nonlinear terms.

\section{Stability calculation}

Stability calculations for differential-integral equations are rarely attempted for obvious reasons. However, by a two parameter expansion, we will be able to sidestep the inherent difficulties. 
In this calculation we expand in both the amplitude $a$ and the wavelength of the perturbation $K$, taken to be of the same order. Thus, from (6):

$$
f_{0}=A\left[1+a \cos (k \xi)+\frac{1}{2} a^{2}+\frac{1}{2} a^{2} \cos (2 k \xi)+\mathrm{O}\left(a^{3}\right)\right]
$$

and

$$
\begin{aligned}
& f=f_{0}+\delta f \\
& \delta f=\mathrm{e}^{\mathrm{i} K \xi+\Gamma t}\left[\Psi_{0}(\xi)+\Psi_{1}(\xi)+\ldots\right]+\text { c.c. }, \\
& \Gamma=\Gamma_{1}+\Gamma_{2}+\ldots
\end{aligned}
$$

and $\Gamma$ is a growth rate. It follows from Floquet's theorem [12], that all $\Psi_{n}$ are periodic with the same period as $f_{0}(\xi)$. This introduces a degeneracy in (14), as $K \rightarrow n k \pm K$ are also admissible. Equation (1) becomes, upon linearization,

$$
\frac{\partial \delta f}{\partial t}-\lambda \frac{\partial \delta f}{\partial \xi}+\alpha \frac{\partial}{\partial \xi}\left(f_{0} \delta f\right)-\beta \frac{\partial^{2}}{\partial \xi^{2}} \mathcal{H}(\delta f)=0 .
$$

The dispersion relation in the linear limit, $\delta f \propto \mathrm{e}^{\mathrm{i}(k x-\omega t)}$ is given by the frequency $\omega=(-\lambda+\alpha A-\beta|k|) k$. For small $a$ we have, from (7) and (8),

$$
\begin{aligned}
& \left(\frac{\beta k}{\lambda}\right)^{2}=1-a^{2}, \\
& \lambda=\beta k\left(1+a^{2} / 2+\ldots\right), \\
& A=\frac{2 \beta k}{\alpha}\left(1-a^{2} / 2+\ldots\right) .
\end{aligned}
$$

Introducing the linear operator $L$ :

$$
L=-\lambda \frac{\partial}{\partial \xi}+\alpha A \frac{\partial}{\partial \xi}-\beta \frac{\partial^{2}}{\partial \xi^{2}} \mathcal{H},
$$

we see that, at zero order in $a$ :

$$
L \Psi_{0}=0, \quad \Psi_{0}=\mathrm{e}^{\mathrm{i}(k \xi+\gamma)} .
$$

However, we find from (16) that there is a second-order correction

$$
L \Psi_{0}=-\frac{3}{2} \mathrm{i} k^{2} \beta a^{2} \mathrm{e}^{\mathrm{i}(k \xi+\gamma)} .
$$

In first order in $a$ and $k$ we find

$$
\begin{aligned}
& L \Psi_{1}=-\left(\Gamma_{1}+\mathrm{i} \omega_{k} k\right) \Psi_{0}-\alpha A a\left[\Psi_{0} \cos (k \xi)\right]_{\xi}, \\
& \omega_{k}=-\lambda .
\end{aligned}
$$

Thus, to avoid secular terms, $\Gamma_{1}=\mathrm{i} \lambda K$. For possible instabilities we must therefore proceed to second order. 
From (20) we find

$$
\Psi_{1}=a \mathrm{e}^{\mathrm{i}(k \xi+\gamma)}+\text { const. }
$$

When we perform the second-order calculation, using (19) and (22) in (15), we find that the constant is $-\frac{1}{2} a \mathrm{e}^{\mathrm{i} \gamma}$ and also that secular terms in $\Psi_{2}$ can only be avoided if $\mathrm{e}^{\mathrm{i}(k \xi+\gamma)}$ and $\mathrm{e}^{-\mathrm{i}(k \xi-\gamma)}$ terms on the right hand side are also zero. We obtain finally

$$
\Gamma_{2}= \pm \mathrm{i} \beta K^{2} .
$$

The stable perturbation splits. This result takes the form of an extended Lighthill theorem [12]. (In the original Lighthill form, the second term under the square root was missing [13]):

$$
\Gamma_{2}= \pm \sqrt{\omega_{a 2} \omega_{k k} a^{2} K^{2}-\omega_{k k}^{2} K^{4} / 4}
$$

(subscripts denote differentiation). Indeed, we see from (15) that $\omega_{k k}= \pm 2 \beta$. Calculation of $\omega_{a^{2}}$, as above, is of course not so straightforward (though all terms cancel). Due to the degeneracy introduced by the periodicity of the $\Psi_{n}$, $\Gamma_{2}(n k \pm K)$ are also roots. Collecting our results, we can write our solution as a Fourier expansion, slightly extended because of the degeneracy

$$
\Omega=\mathrm{i} \Gamma=\omega_{k} K \pm \frac{1}{2} \omega_{k k} K^{2}
$$

The amplitude of the nonlinear wave only introduces the degeneracy as mentioned above. Exceptionally for this kind of problem, the amplitude does not appear in the nonlinear dispersion relation as calculated up to second order. This is a rare example of Lighthill's $\omega_{a^{2}}$ vanishing.

A different method, such that we expand in $K$ only, keeping the wave profile exact [12], just yields agreement to lowest order

$$
\Gamma_{1}=\mathrm{i} \lambda K
$$

missing the splitting. Ironically, this calculation, though ostensibly yielding less information than the above, is much more complicated (see the first reference of [12], Chapter 8 for the method). However, it does tell us that there are no contributions like $a^{2} K$ to $\Omega$.

\section{Summary}

We have been able to resolve the question of the stability of known, exact nonlinear wave solutions to the Benjamin-Ono (BO) equation. Historically, first its integrability was established, e.g. by the ubiquitous Painlevé method [4], then, almost simultaneously, $N$ soliton solutions were constructed and investigated for stability [8]. Thus it was time to determine the question of stability of nonlinear waves and derive Lighthill's formula for perturbations to them. This step has 
now been taken for this generic equation. The fact that Lighthill's formula can be derived for perturbed nonlocal equations, as well as the procedure outlined here, may prove useful in other contexts. These could include extensions to other members of the BO family, either with an extra interaction term [3], or else a couple of BO equations describing two interacting wave trains at different depths [14]. These extensions should be straightforward but could be laborious.

In all these cases, both treated here and indicated immediately above, stability must be established before wave solutions can be considered to be of physical significance, rather than just mathematical artifacts. It is a strange sign of the times that wave dynamics often lags behind soliton work, even though nonlinear wave trains are more common in everyday life.

\section{Acknowledgments}

The authors would like to thank Dr A.A. Skorupski for help in writing this paper. This work was partially supported by grant No. 2P03B09722 of the State Committee for Scientific Research.

\section{Appendix}

Equation (12) has exact solutions that represent propagating waves [2, 14]:

$$
\begin{aligned}
& f(\xi)=f_{0}-\frac{B}{1-b \cos (k \xi)}, \quad \xi=x-\lambda t, \\
& B=3 \beta k^{2} / f_{0}, \\
& \lambda=f_{0}^{2}-\beta k^{2}, \\
& b=\sqrt{1-B /\left(2 f_{0}\right)},
\end{aligned}
$$

where now $f_{0}$ is a constant.

When a small $b$, small $K$ analysis, similar to that of this paper, is performed, we obtain (with the same notation)

$$
\frac{\partial \delta f}{\partial \tau}+\beta k^{2} \frac{\partial}{\partial \xi}\left[\left(1+6 b \cos k \xi+6 b^{2} \cos 2 k \xi+\ldots\right) \delta f\right]+\beta \frac{\partial^{3} \delta f}{\partial \xi 3}=0 .
$$

We find that

$$
\Psi_{0}=\cos (k \xi+\gamma)
$$

and next, in first order,

$$
\Psi_{1}=b \cos (2 k \xi+\gamma)-3 b \cos \gamma
$$

In second order, exclusion of secular terms, produced by $\cos (k \xi+\gamma)$ and $\sin (k \xi+\gamma)$ on the right, leads to the condition 


$$
\Gamma_{2}= \pm \mathrm{i} \sqrt{36 \beta k^{2} b^{2} K^{2}+9 \beta^{2} k^{2} K^{4}}
$$

Equation (A.8) is likewise in the form of an extended Lighthill theorem, as $\omega_{k k}=-6 \beta k$. Now $\omega_{a^{2}} \omega_{k k}$ is negative and stability is obtained again (compare Eq. (A.8) with (24)).

The Lorentzian is obtained in the $k \rightarrow 0$ limit

$$
f=f_{0}-\frac{4 \delta^{2} f_{0}}{\delta^{2}+\xi^{2} f_{0}}, \quad \delta^{2}=\frac{3 \beta}{2 f_{0}}, \quad \lambda=f_{0}^{2},
$$

and is stable, as can be seen from (A.8) when $k=0$. The stability of the algebraic soliton is important in several branches of physics, including not only situations described here (for an optical context see [15]).

This stability calculation is not included in that of [11], for reasons explained in the previous section.

\section{References}

[1] T.E. Benjamin, J. Fluid Mech. 29, 559 (1967); R.A. Davis, A. Acrivos, J. Fluid Mech. 29, 593 (1967).

[2] H. Ono, J. Phys. Soc. Jpn. 39, 1082 (1975).

[3] B. Fuchssteiner, T. Schultze, Phys. Lett. A 204, 336 (1995).

[4] K.M.T. Tamizhmani, A. Ramani, B.J. Grammaticos, J. Phys. A 30, 1017 (1997).

[5] Y.J. Matsumo, J. Phys. A 12, 125 (1979).

[6] Y.J. Matsumo, Phys. Soc. Jpn. 57, 1924 (1988).

[7] T. Miloh, M. Prestin, L. Shtilman, M.P. Tulin, Wave Motion 17, 11 (1993).

[8] Y. Matsumo, D.J. Kaup, J. Math. Phys. 38, 5195 (1997).

[9] D.J. Kaup, T.I. Lakoba, Y. Matsumo, Inverse Problems 15, 215 (1999).

[10] B. Fornberg, G.B. Whitham, Philos. Trans. R. Soc. Lond. A 289, 373 (1978).

[11] K. Murawski, E. Infeld, Aust. J. Phys. 41, 1 (1988).

[12] E. Infeld, G. Rowlands, Nonlinear Waves, Solitons and Chaos, 2nd ed., Cambridge University Press, Cambridge, UK 2000, Chapter 5; E. Infeld, G. Rowlands, A. Senatorski, Proc. R. Soc. Lond. A 455, 4363 (1999); E. Infeld, A.A. Skorupski, G. Rowlands, ibid. 458, 1231 (2002).

[13] G.B. Whitham, Linear and Nonlinear Waves, Wiley, New York 1974.

[14] T.L. Perelman, A.K. Friedman, M.M. Elyashvich, Phys. Lett. A 47, 321 (1974).

[15] E.J. Hanamura, Phys. Soc. Jpn. 37, 1598 (1974). 